\title{
Bone Marrow Necrosis in a Child With Sepsis-Induced Macrophage Activation Syndrome
}

\author{
Biraj Parajulia, Jeyaraj Munusamya , Arun K. Baranwala, c, \\ Prashant Sharma ${ }^{\mathrm{b}}$, Muralidharan Jayashree ${ }^{\mathrm{a}}$
}

\begin{abstract}
Bone marrow necrosis (BMN) is a rare condition, mostly described with hematological malignancies. Association of BMN with sepsisinduced macrophage activation syndrome (MAS) is rarely reported in the literature. Recently, we managed a 10-year-old girl with Staphylococcus aureus sepsis and MAS who had extensive BMN. Patient initially improved with antibiotics, source control, supportive care and immunomodulatory therapies (intravenous immunoglobulin and methylprednisolone). However, she succumbed to transfusion-related acute lung injury. Sepsis, MAS and BMN seem to represent increasing levels of immune dysregulation, hypercytokinemia and hyperinflammation.
\end{abstract}

Keywords: Sepsis; Macrophage activation syndrome; Bone marrow necrosis; Myelonecrosis

\section{Introduction}

Bone marrow necrosis $(\mathrm{BMN})$ is a rare condition, and most commonly described with hematological malignancies [1]. Isolated infections are rarely reported to cause BMN. We report extensive BMN in an apparently immunocompetent child with disseminated methicillin-resistant Staphylococcus aureus (MRSA) disease with sepsis-induced macrophage activation syndrome (MAS). This case is presented to emphasize the routine search for MAS in sepsis patients with pancytopenia and hyperferritinemia. It may improve the outcome with early immunomodulatory therapy. To best of our knowledge, this is the first case of BMN in a child with documented sepsis-induced MAS.

Manuscript submitted September 12, 2019, accepted January 7, 2020

aDepartment of Pediatrics, Advanced Pediatrics Centre, Postgraduate Institute of Medical Education and Research, Chandigarh 160012, India

bepartment of Hematology, Advanced Pediatrics Centre, Postgraduate Institute of Medical Education and Research, Chandigarh 160012, India

${ }^{\mathrm{c} C o r r e s p o n d i n g ~ A u t h o r: ~ A r u n ~ K . ~ B a r a n w a l, ~ D i v i s i o n ~ o f ~ P e d i a t r i c ~ C r i t i c a l ~}$ Care and In-charge, Pediatric Cardiac Care Unit, Advanced Pediatrics Centre, PGIMER, Chandigarh160012, India. Email: baranwal1970@gmail.com

doi: https://doi.org/10.14740/ijcp346

\section{Case Report}

A 10-year-old girl was referred with high-grade intermittent fever $\left(39.4{ }^{\circ} \mathrm{C}\right)$ for 10 days, and with respiratory distress and septic shock at presentation. On the second day of fever, she developed erythematous rash over legs, arms and trunk. Four days later, she had an episode of epistaxis, pain and swelling in bilateral knee and hip joints. There was no history of diarrhea, hematemesis, icterus, melena or burning micturition. She was treated with intravenous antibiotics (amikacin and levofloxacin), fluids, and transfusion of platelets and packed red blood cells $(\mathrm{RBCs})$ for thrombocytopenia $\left(31 \times 10^{9} / \mathrm{L}\right)$ and anemia (hemoglobin $74 \mathrm{~g} / \mathrm{L}$ ) at the referring hospital. Parents denied any previous hospitalizations or similar episode in the child and the family. She was optimally immunized as per India's National Immunization Schedule. She was born to non-consanguineous parents of lower middle class. Examination revealed pallor, bilateral cervical and axillary lymphadenopathy, bilateral pitting pedal edema, blanchable erythematous rash all over the body, bilateral pulmonary crepitations, hepatomegaly $(6 \mathrm{~cm}$ below the right costal margin) and an abscess $(5 \times 5$ $\mathrm{cm})$ over the right chest wall. She had respiratory failure with shock at the time of presentation. However, her sensorium was normal with no focal neurological deficit.

She needed inotropic support and ventilation for septic shock (bounding pulses, tachycardia, blood pressure at the fifth centile, capillary refill $<2$ s) and hypoxic respiratory failure. Chest radiograph showed bronchopneumonia and bilateral pleural effusion. In view of chest abscess and local epidemiology, a possibility of staphylococcal infection with toxic shock syndrome was considered. Laboratory findings are summarised in Table 1. Investigations revealed pancytopenia, deranged coagulogram and nonoliguric acute kidney injury. Serum ferritin, triglycerides, fibrinogen and D-dimer were elevated. Joint imaging did not reveal any effusion. MRSA grew in blood and pus from chest wall abscess. Bone marrow aspiration revealed hemophagocytosis and extensive BMN with very few viable hematopoietic elements (Fig. 1a-c). No abnormal cells or infectious organisms were seen. Ziehl-Neelsen stain for acid fast bacilli and stains for fungi were negative (Fig. $1 \mathrm{~d}$, e). Serum sample taken before starting immunomodulatory therapy revealed elevated soluble cluster of differentiation CD25 (12,711 ng/L; normal, 458 - 1,997 ng/L) and CD163 $(2,794.8 \mu \mathrm{g} / \mathrm{L}$; normal, 88 - $902 \mu \mathrm{g} / \mathrm{L})$ (Quantikine ${ }^{\circledR}$ Enzymelinked Immunosorbent Assay kit from R\&D Systems ${ }^{\circledR}$, Min- 
Table 1. Laboratory Parameters During the Hospital Course

\begin{tabular}{|c|c|c|c|c|}
\hline Investigations & At admission & Day 3 & Day 7 & Day 10 \\
\hline \multicolumn{5}{|l|}{ Complete blood counts } \\
\hline Hemoglobin (g/L) & 74 & 108 & 95 & 61 \\
\hline Neutrophils (\%) & - & 7.6 & 2.9 & 84.7 \\
\hline Lymphocytes (\%) & - & 87.2 & 93.4 & 11.4 \\
\hline Eosinophils (\%) & - & - & 0.8 & - \\
\hline Basophils (\%) & - & - & - & - \\
\hline Platelets $\left(\times 10^{9} / \mathrm{L}\right)$ & 38,000 & 4,000 & 28,000 & 53,000 \\
\hline \multicolumn{5}{|l|}{ Renal function test } \\
\hline Urea (mg/dL) & 65 & 108 & 96 & 107 \\
\hline PTI (\%) & 62 & 66 & 76 & \\
\hline INR & 2.53 & 1.53 & 1.33 & \\
\hline aPTT (s) & 37 & & & \\
\hline D-dimer (ng/mL) & & 5090 & 4741 & \\
\hline \multicolumn{5}{|l|}{ MAS parameters } \\
\hline Ferritin $(\mathrm{ng} / \mathrm{mL})$ & 2,623 & & 3,245 & \\
\hline Fibrinogen $(\mathrm{g} / \mathrm{L})$ & & 5.72 & 3.41 & \\
\hline Triglycerides (mg/dL) & & 266 & 156 & \\
\hline \multicolumn{5}{|l|}{ Inflammatory parameters } \\
\hline \multicolumn{5}{|l|}{ Microbiological investigations } \\
\hline \multirow[t]{2}{*}{ Blood culture } & \multicolumn{4}{|c|}{ Day 1: methicillin-resistant Staphylococcus aureus } \\
\hline & \multicolumn{4}{|c|}{ Day 8: Candida tropicalis } \\
\hline Pus culture & \multicolumn{4}{|c|}{ Day 2: methicillin-resistant Staphylococcus aureus } \\
\hline
\end{tabular}

PT: prothrombin time; INR: international normalized ratio; aPTT: activated partial thromboplastin time; CRP: C-reactive protein.

neapolis, USA). These were done as part of an ongoing research project.

Intravenous ceftriaxone $(50 \mathrm{mg} / \mathrm{kg} /$ dose $\mathrm{q} 12 \mathrm{~h})$, vancomy$\operatorname{cin}(20 \mathrm{mg} / \mathrm{kg} /$ dose $\mathrm{q} 8 \mathrm{~h})$ and clindamycin $(10 \mathrm{mg} / \mathrm{kg} /$ dose $\mathrm{q} 8 \mathrm{~h})$ were initiated at admission. Ceftriaxone was stopped after receiving the culture report, while clindamycin was continued for 5 days. Vancomycin was continued till demise. Chest wall abscess was drained. Intravenous immunoglobulin (IVIG) (2 $\mathrm{g} / \mathrm{kg}$ ) was given on day 4 for hyperferritinemic sepsis and suspected MAS. On day 6, intravenous pulse methylprednisolone (30 mg/kg/day for 3 days) was given for deteriorating platelet count. Subsequently, leukocyte and platelet count started improving (Fig. 1g). She could be weaned off inotropes (maximum vasoactive inotrope score, 30 ), and was requiring mini- mal ventilator support. She received multiple blood cell and platelet transfusions during this period. Her general condition started improving. Her stay was punctuated by central lineassociated blood stream infection with Escherichia coli (E. coli) (day 6) and primary blood stream infection with Candida tropicalis (C. tropicalis) (day 8), (Table 1), which were managed with imipenem (25 mg/kg q6h) and liposomal amphotericin B $(5 \mathrm{mg} / \mathrm{kg} /$ day $)$.

On day 9, platelet and packed RBCs were transfused for anemia and thrombocytopenia with clinical bleeding. After 4 - $5 \mathrm{~h}$, she developed bilateral crepitations and desaturation. Her ventilator requirement increased significantly, and later she required high frequency oscillation (oxygenation index, 50; chest radiograph, extensive bilateral infiltrates (Fig. 1f)). 

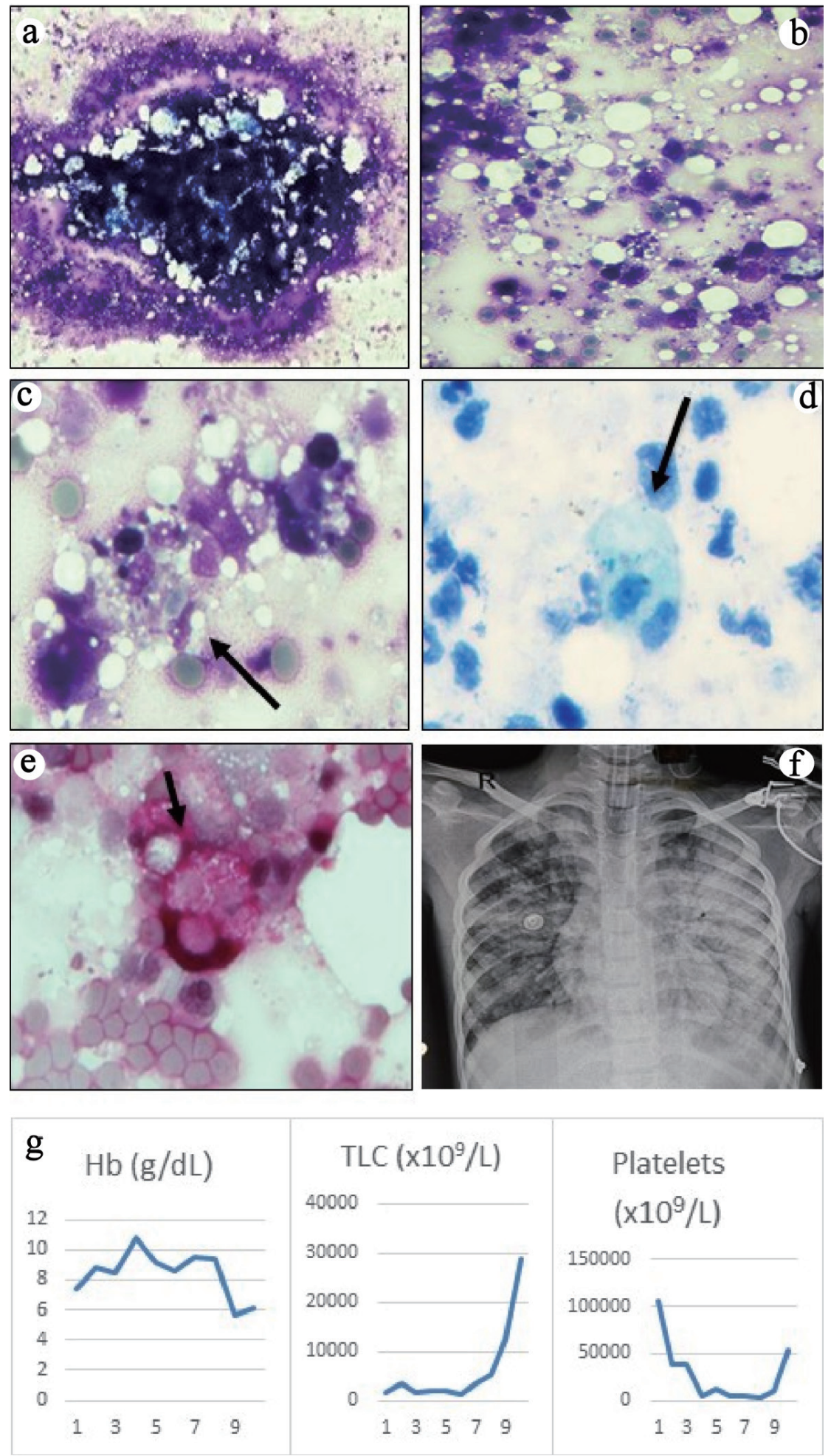

Figure 1. (a) Bone marrow aspirate smears were particulate and appeared normocellular (May-Grunwald Giemsa, $\times 40$ ). (b) The background showed mostly necrotic cells with ill-defined cellular boundaries admixed with fat and very few viable cells (MayGrunwald Giemsa, × 200). (c) High power examination revealed several histiocytes with ingested erythrocytes, lymphocytes and cell debris (May-Grunwald Giemsa, $\times 400$ ). Ziehl-Neelsen $(Z N)$ stain for acid-fast bacilli (d) and periodic acid-Schiff stain for fungi (e) were negative, but highlighted the phagocytic histiocytes $(\times 400$, ZN stain counterstained with methylene blue, PAS stain counterstained with hematoxylin). (f) Chest radiograph showed bilateral pulmonary edema secondary to transfusion-related lung injury. (g) Graphs showing trends of the blood counts during hospital stay. 
Table 2. Clinical Details of Children With Infection-Induced Bone Marrow Necrosis Reported in Literature

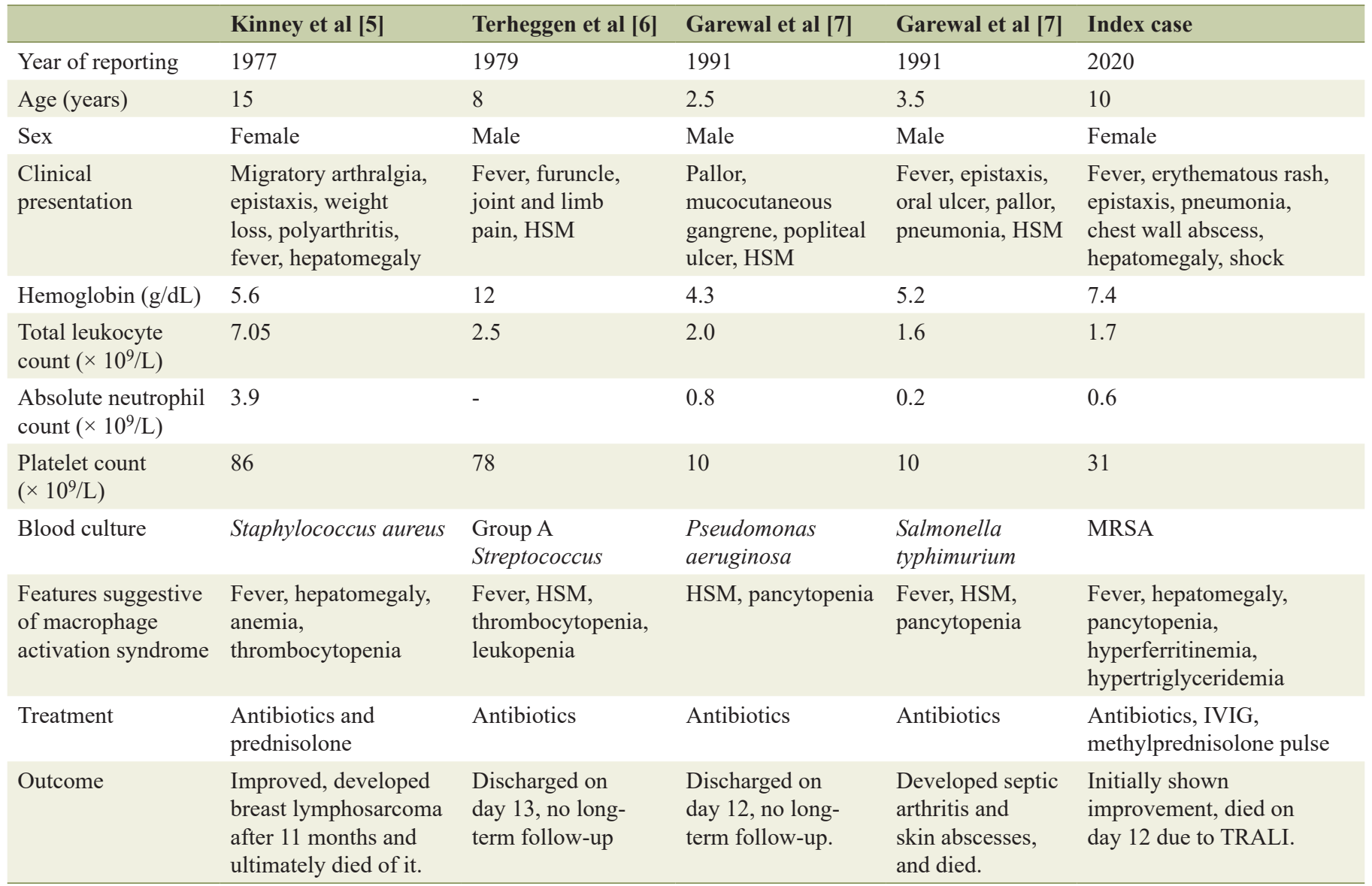

HSM: hepatosplenomegaly; MRSA: methicillin-resistant Staphylococcus aureus; IVIG: intravenous immunoglobulin; TRALI: transfusion-related acute lung injury.

Shock resurfaced and remained refractory despite vasoactiveinotropic support. Three days later, she succumbed to multiorgan failure. Autopsy could not be done for lack of parental consent.

\section{Discussion}

We described a child with MRSA sepsis with toxic shock syndrome who continued to deteriorate and develop multiple organ dysfunction syndrome (MODS) despite receiving appropriate antibiotics, source control and supportive care. MAS was suspected in view of persistent pancytopenia, hyperferritinemia and hypertriglyceridemia. With immunomodulatory therapies (intravenous immunoglobulin and methylprednisolone), she initially showed appreciable clinical improvement. However, she succumbed to transfusion-related acute lung injury (TRALI) shortly after. To best of our knowledge, this is the first case of BMN in a child with culture-positive sepsisinduced MAS.

Sepsis is a state of infection-induced dysregulated innate and adaptive immunity leading to hypercytokinemia, inflammation and persistent multi-organ failure $[2,3]$. Interferon- gamma activates macrophages resulting in MAS [3]. BMN is defined as necrosis of myeloid tissue and stroma in $>50 \%$ of parenchyma without cortical bone involvement. BMN is considered to be caused by cellular hypoxia, endothelial and vascular injury, endotoxins, cytokines, and oxygen free radicals [1]. Both MAS and BMN are associated with poor prognosis $[1,3]$. With uncontrolled hyperinflammation associated with sepsis, it appears logical for MAS to progress on to BMN. We could find only one report of such association in a 32-year-old male patient with Bacteroides fragilis abscess and MAS [4].

Sepsis-induced BMN is extremely rare; a handful of cases have been reported. Gram-negative (E. coli, Salmonella, Pseudomonas aeruginosa, Citrobacter freundii, Bacteroides fragilis) and Gram-positive (Streptococcus, Staphylococcus) bacteria, Coxiella burnetii, tuberculosis, mucormycosis and parvovirus have been reported to cause BMN in otherwise normal patients $[1,4]$. However, literature search revealed only one adult patient in whom defining features of MAS could be demonstrated including hemophagocytosis in the bone marrow [4].

We could find only four pediatric cases [5-7] of BMN associated with sepsis; all four were reported in the later part of 
the last century. Table 2 [5-7] provides comparison of these previously reported cases with the index case. All these four patients had hepatosplenomegaly and pancytopenia. It is possible that they all might have sepsis-induced MAS which remained undiagnosed for the lack of awareness about MAS at that time. Three of them improved with antibiotics and supportive care, while the fourth one died. The patient reported by Kinney et al [5] who received steroid during acute phase, died of breast lymphosarcoma on follow-up. The index patient satisfied the diagnostic criteria of MAS as she had persistent fever, pancytopenia, hepatomegaly, hyperferritinemia, hypertriglyceridemia, bone marrow hemophagocytosis, significantly elevated soluble CD25 and CD163 levels [3]. Sepsis-induced MAS with exaggerated hemophagocytosis is the likely explanation for the observed extensive BMN in the index case as well as in the previously reported cases. An autopsy case series reported extensive BMN in two girls (aged 9 and 18 years respectively) due to paracoccidioidomycosis [8]; however there is no information if they had hemophagocytosis. Leukoerythroblastic picture on peripheral blood smear, hyperuricemia, elevated transaminases, lactate dehydrogenase (LDH) and alkaline phosphatase are also reported [2]; however none of these were present in the index case.

BMN is usually rapidly fatal. In some, prolonged injury and suboptimal tissue recovery may result in bone marrow fibrosis [1]. Among survivors, necrotic cells get cleared by phagocytosis followed by replenishment of normal bone marrow tissue. Thus, management for sepsis-induced hyperinflammatory states should include early antibiotic treatment, source control, optimal support for multiorgan dysfunction, high index of suspicion for MAS and prompt immunomodulatory therapy to help recover from MAS. Presence of hyperferritinemia may help identify high-risk patients with likelihood of having MAS. Recommended immunotherapies include IVIG, methylprednisolone, plasmapheresis and interleukin-1 (IL-1) receptor antagonist [3]. The index case revealed initial improvement with antibiotics, source control, IVIG and pulse methylprednisolone as shown by recovery in cardiovascular, respiratory, renal and hematological parameters. However, healthcare-associated infections with $E$. coli and $C$. tropicalis might have caused a second hit with continued hyperinflammation. Though identifying $\mathrm{BMN}$ in a patient with sepsis-induced MAS will not alter the case management, it will affect the prognosis. The pre-terminal development of severe acute respiratory distress syndrome shortly after transfusion of platelets and packed RBCs was probably due to TRALI. Hyperinflammatory state, as in the index case, is a well-known predisposing factor [9]. Pulmonary embolization of fat and necrotic bone marrow tissues may also be possible [1]; however improvement in clinical condition and hematological parameters precludes it. An autopsy could have clarified the pre-terminal events better.

\section{Conclusions}

Sepsis, MAS and BMN probably represent different points on the spectrum of increasing levels of immune dysregulation and hyperinflammation. MAS may specifically be looked for in the select group of septic shock patients with pancytopenia, MODS and hyperferritinemia. It may help institute early immunomodulatory therapy and better clinical outcome. Blood products should be judiciously used in patients with hypercytokinemia/ hyperinflammation to help avoid TRALI.

\section{Acknowledgments}

None to declare.

\section{Financial Disclosure}

None to declare.

\section{Conflict of Interest}

On behalf of all authors, the corresponding author states that there is no conflict of interest.

\section{Informed Consent}

Parent's consent for publication of this case report has been obtained.

\section{Author Contributions}

BP, JM, PS and AKB managed the patient. JM did literature review. BP and JM prepared the first draft. AKB finalised the draft, and will act as guarantor. M J critically reviewed the manuscript.

\section{Data Availability}

On behalf of all authors, the corresponding author declares that data supporting the findings are available within the article.

\section{References}

1. Cabral TCS, Fernandes CM, Lage LAC, Zerbini MC, Pereira J. Bone marrow necrosis: literature review. J Bras Patol Med Lab. 2016; 52:182-188.

2. Delano MJ, Ward PA. Sepsis-induced immune dysfunction: can immune therapies reduce mortality? J Clin Invest. 2016;126:23-31.

3. Castillo L, Carcillo J. Secondary hemophagocytic lymphohistiocytosis and severe sepsis/ systemic inflammatory response syndrome/multiorgan dysfunction syndrome/ macrophage activation syndrome share common intermediate phenotypes on a spectrum of inflammation. Pediatr Crit Care Med. 2009;10(3):387-392.

4. Takahashi T, Rykken J. Progressive Diffuse Osteonecro- 
sis in a Patient with Secondary Hemophagocytic Lymphohistiocytosis. Case Rep Radiol. 2015;2015:730719.

5. Kinney TR, Koch PA, Gottlieb RP, Donaldson MH. Bone marrow necrosis in children: three instances. Clin Pediatr (Phila). 1977;16(6):565-568.

6. Terheggen HG, Lampert F. Acute bone marrow necrosis caused by streptococcal infection. Eur J Pediatr. 1979;130(1):53-58.

7. Garewal G, Marwaha N, Marwaha RK, Das KC. Bone marrow necrosis and pancytopenia associated with gram negative septicemia. Indian Pediatr. 1991;28(1):79-81.

8. Resende LS, Mendes RP, Bacchi MM, Marques SA, Barraviera B, Souza LR, Meira DA, et al. Bone marrow necrosis related to paracoccidioidomycosis: the first eight cases identified at autopsy. Histopathology. 2009;54(4):486-489.

9. Vlaar APJ, Toy P, Fung M, Looney MR, Juffermans NP, Bux J, Bolton-Maggs $\mathrm{P}$, et al. A consensus redefinition of transfusion-related acute lung injury. Transfusion. 2019;59(7):2465-2476. 